Available online at www.qu.edu.iq/journalcm

JOURNAL OF AL=QADISIYAH FOR COMPUTER SCIENCE AND MATHEMATICS

ISSN:2521-3504(online) ISSN:2074-0204(print)

\title{
Some Results on Symmetric Reverse *-n-Derivations
}

\section{Anwar Khaleel Faraj ${ }^{a}$, Ruqaya Saadi Hashem $^{b}$}

a Department of Applied Sciences, University of Technology, Baghdad, Iraq. Email : anwar_78_2004@yahoo.com

${ }^{b}$ Department of Applied Sciences , University of Technology, Baghdad, Iraq. Email : ruqaya.saadi94@gmail.com

\section{A R T ICLE INFO}

\section{Article history:}

Received: $01 / 04 / 2019$

Rrevised form: 18 /04/2019

Accepted : $23 / 04 / 2019$

Available online: 30 /05/2019

Keywords:

Prime ring, $*$ - $n$-derivation, Reverse

*-n-derivation, Commuting

mapping, Centralizing mapping,

Permuting mapping.

\section{A B S T RAC T}

In this paper, the commuting and centralizing of symmetric reverse $*$ - $n$-derivation on Lie ideal are studied and the commutativity of prime $*$-ring with the concept of symmetric reverse $*-n$-derivations are proved under certain conditions.

MSC: 13N15

Corresponding author Ruqaya Saadi Hashem

Email addresses: ruqaya.saadi94@gmail.com 


\section{Introduction}

Throughout this paper $\mathcal{R}$ will represent an associative ring with center $\mathcal{Z}(\mathcal{R})$. For any $v, \gamma \in \mathcal{R}$, the commutator $v \gamma-\gamma v$ was denoted by $[v, \gamma]$ and the anti-commutator $v \circ \gamma$ was denoted by $v \gamma+\gamma v$ [8]. A ring $\mathcal{R}$ is said to be $n$-torsion free if $n a=0$ with $a \in \mathcal{R}$ then $a=0$, where $n$ is nonzero integer [7]. Recall that a ring $\mathcal{R}$ is said to be prime if $a \mathcal{R} b=0$ implies that either $a=0$ or $b=0$ for all $a, b \in \mathcal{R}$ [12] and it is semiprime if $a \mathcal{R} a=0$ implies that $a=0$ for all $a \in \mathcal{R}$ [7]. An additive mapping $\xi: \mathcal{R} \rightarrow \mathcal{R}$ is called a derivation if $\xi(v \gamma)=\xi(v) \gamma+v \xi(\gamma)$ for all $v, \gamma \in \mathcal{R}$ [11]. In [2] were introduced the concept of reverse derivations; an additive mapping $\xi: \mathcal{R} \rightarrow \mathcal{R}$ is called a reverse derivation if $\xi(v \gamma)=\xi(\gamma) v+\gamma \xi(v)$ for all $v, \gamma \in \mathcal{R}$. A map $\mathcal{F}: \mathcal{R} \rightarrow \mathcal{R}$ is said to be commuting (resp. centralizing) on $\mathcal{R}$ if $[\mathcal{F}(v), v]=0$ (resp. $[\mathcal{F}(v), v] \in \mathcal{Z}(\mathcal{R})$ ) for all $v \in \mathcal{R}$ [12]. An additive mapping $v \rightarrow v^{*}$ of $\mathcal{R}$ into itself is called an involution if the following conditions are satisfied (i) $(v \gamma)^{*}=\gamma^{*} v^{*}$ (ii) $\left(v^{*}\right)^{*}=v$ for all $v, \gamma \in \mathcal{R}$ [8]. A ring equipped with an involution is known as ring with involution or $*$-ring. Let $\mathcal{R}$ be a $*$-ring. An additive mapping $\xi: \mathcal{R} \rightarrow \mathcal{R}$ is called a $*$-derivation (resp. a reverse $*$-derivation) if $\xi(v \gamma)=\xi(v) \gamma^{*}+v \xi(\gamma)$ (resp. $\left.\xi(v \gamma)=\xi(\gamma) v^{*}+\gamma \xi(v)\right)$ for all $v, \gamma \in \mathcal{R}$ [2]. An additive subgroup $\mathcal{U}$ of $\mathcal{R}$ is called Lie ideal if whenever $u \in \mathcal{U}$, $\mathfrak{r} \in \mathcal{R}$ then $[u, \mathfrak{r}] \in \mathcal{U}$ [7]. A Lie ideal $\mathcal{U}$ of $\mathcal{R}$ is called a square closed Lie ideal of $\mathcal{R}$ if $u^{2} \in \mathcal{U}$, for all $u \in \mathcal{U}$ [3]. A square closed Lie ideal $\mathcal{U}$ of $\mathcal{R}$ such that $\mathcal{U} \nsubseteq \mathcal{Z}(\mathcal{R})$ is called an admissible Lie ideal of $\mathcal{R}$ [11]. Relationship between derivations and reverse derivations with examples were given by [13]. Recently there has been a great deal of work done by many authors on commuting and centralizing mappings on prime rings and semiprime rings, see ([4],[5],[6],[9],[10]). In [2] studied the notion of a $*$-derivation of $\mathcal{R}$. Recently [1] defined the concept of $*$ - $n$-derivation in prime $*$-rings and semiprime $*$-rings. Many authors have proved the commutativity of prime and semiprime rings admitting derivation ([11],[3]). In the present paper the commuting and centralizing of symmetric reverse $*$-n-derivation of Lie ideal are studied under certain conditions and on the other hand the commutativity of prime $*$-ring with symmetric reverse $*$ - $n$-derivations that satisfying certain identities and some regarding results have also been discussed. Throughout this paper consider $n$ is a fixed positive integer. 


\section{Preliminaries}

Some definitions and fundamental facts of symmetric reverse $*$-n-derivations are recalled in this section, which are principals of reverse left $*$-n-derivation.

\section{Proposition (2.1) [8]}

Let $\mathcal{R}$ be a ring, then for all $v, \gamma, z \in \mathcal{R}$ we have

$1-[v, \gamma z]=\gamma[v, z]+[v, \gamma] z$

2- $\quad[v \gamma, z]=v[\gamma, z]+[v, z] \gamma$

3- $\quad v \circ(\gamma z)=(v \circ \gamma) z-\gamma[v, z]=\gamma(v \circ z)+[v, \gamma] z$

4- $(v \gamma) \circ z=v(\gamma \circ z)-[v, z] \gamma=(v \circ z) \gamma+v[\gamma, z]$

\section{Definition (2.2) [9]}

A map $\xi: \mathcal{R}^{n} \rightarrow \mathcal{R}$ is called permuting (or symmetric) if the equation $\xi\left(v_{1}, v_{2}, \ldots, v_{n}\right)=\xi\left(v_{\pi(1)}, v_{\pi(2)}, \ldots, v_{\pi(n)}\right)$ holds, for all $v_{i} \in \mathcal{R}$ and for every permutation $\{\pi(1), \pi(2), \ldots, \pi(n)\}$.

\section{Definition (2.3) [9]}

A map $\delta: \mathcal{R} \rightarrow \mathcal{R}$ is define as $\delta(v)=\Omega(v, v, \ldots, v)$ for all $v \in \mathcal{R}$, where $\Omega: \mathcal{R}^{n} \rightarrow \mathcal{R}$ is called the trace of the symmetric mapping $\Omega$.

It is clear that the trace function $\delta$ is an odd function if $n$ is an odd number and is an even function if $n$ is an even number.

\section{Note (2.4) [9]}

Let $\delta$ be a trace of an $n$-additive symmetric map $\delta: \mathcal{R}^{n} \rightarrow \mathcal{R}$, then $\delta$ satisfies the relation $\delta(\mathrm{v}+\gamma)=\delta(v)+\delta(\gamma)+\sum_{k=1}^{n-1}\left(\begin{array}{l}n \\ k\end{array}\right) h_{k}(\mathrm{v}, \gamma)$ for all $v, \gamma \in \mathcal{R}$ such that $h_{k}(\mathrm{v}, \gamma)=\Omega(\mathrm{v}, \mathrm{v}, \ldots, \mathrm{v}, \gamma, \gamma, \ldots, \gamma)$ where $v$ appears $(n-k)-$ times and $\gamma$ appear $k$-times and $\left(\begin{array}{l}n \\ k\end{array}\right)=\frac{n !}{k !(n-k) !}$.

\section{Definition (2.5) [9]}

An $n$-additive mapping $\xi: \mathcal{R}^{n} \rightarrow \mathcal{R}$ is said to be a symmetric $*$-n-derivation if the following equations are identical: $\xi\left(v_{1} \gamma, v_{2}, \ldots, v_{n}\right)=\xi\left(v_{1}, v_{2}, \ldots, v_{n}\right) \gamma^{*}+v_{1} \xi\left(\gamma, v_{2}, \ldots, v_{n}\right)$

$\xi\left(v_{1}, v_{2} \gamma, \ldots, v_{n}\right)=\xi\left(v_{1}, v_{2}, \ldots, v_{n}\right) \gamma^{*}+v_{2} \xi\left(v_{1}, \gamma, \ldots, v_{n}\right)$

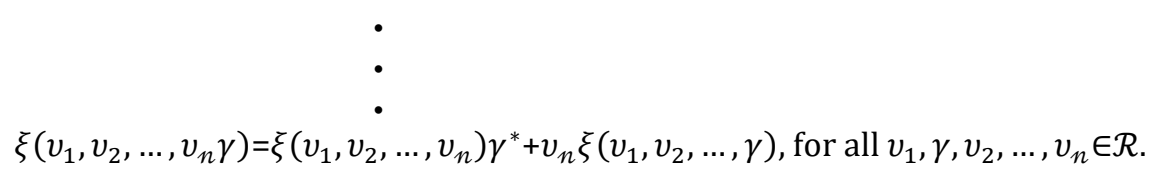

\section{Definition (2.6) [15]}

An $n$-additive symmetric mapping $\xi: \mathcal{R}^{n} \rightarrow \mathcal{R}$ is said to be a symmetric reverse $*-n$-derivation if

$$
\begin{gathered}
\xi\left(v_{1} \gamma, v_{2}, \ldots, v_{n}\right)=\xi\left(\gamma, v_{2}, \ldots, v_{n}\right) v_{1}{ }^{*}+\gamma \xi\left(v_{1}, v_{2}, \ldots, v_{n}\right) \\
\xi\left(v_{1}, v_{2} \gamma, \ldots, v_{n}\right)=\xi\left(v_{1}, \gamma, \ldots, v_{n}\right) v_{2}{ }^{*}+\gamma \xi\left(v_{1}, v_{2}, \ldots, v_{n}\right) \\
\text { • } \\
\dot{\cdot}\left(v_{1}, v_{2}, \ldots, v_{n} \gamma\right)=\xi\left(v_{1}, v_{2}, \ldots, \gamma\right) v_{n}{ }^{*}+\gamma \xi\left(v_{1}, v_{2}, \ldots, v_{n}\right), \text { for all } v_{1} \gamma, v_{2}, \ldots, v_{n} \in \mathcal{R} .
\end{gathered}
$$




\section{Example (2.7):}

Consider $\mathcal{R}=\left\{\left(\begin{array}{lll}0 & a & b \\ 0 & 0 & c \\ 0 & 0 & 0\end{array}\right) \mid a, b, c \in \mathbb{C}\right\}$, where $\mathbb{C}$ is a ring of complex numbers and $\mathcal{R}$ is a non-commutative ring under the usual addition and multiplication of matrices. A map $\xi: \mathcal{R}^{n} \rightarrow \mathcal{R}$ is define by $\xi$

$\left(\left(\begin{array}{ccc}0 & a_{1} & b_{1} \\ 0 & 0 & c_{1} \\ 0 & 0 & 0\end{array}\right),\left(\begin{array}{ccc}0 & a_{2} & b_{2} \\ 0 & 0 & c_{2} \\ 0 & 0 & 0\end{array}\right), \ldots,\left(\begin{array}{ccc}0 & a_{n} & b_{n} \\ 0 & 0 & c_{n} \\ 0 & 0 & 0\end{array}\right)\right)=\left(\begin{array}{ccc}0 & 0 & c_{1} c_{2} \ldots c_{n} \\ 0 & 0 & 0 \\ 0 & 0 & 0\end{array}\right)$, for all

$\left(\begin{array}{ccc}0 & a_{1} & b_{1} \\ 0 & 0 & c_{1} \\ 0 & 0 & 0\end{array}\right),\left(\begin{array}{ccc}0 & a_{2} & b_{2} \\ 0 & 0 & c_{2} \\ 0 & 0 & 0\end{array}\right), \ldots,\left(\begin{array}{ccc}0 & a_{n} & b_{n} \\ 0 & 0 & c_{n} \\ 0 & 0 & 0\end{array}\right) \in \mathcal{R}$.

And $*$ is defined by $\left(\begin{array}{ccc}0 & a & b \\ 0 & 0 & c \\ 0 & 0 & 0\end{array}\right)^{*}=\left(\begin{array}{ccc}0 & c & b \\ 0 & 0 & a \\ 0 & 0 & 0\end{array}\right)$. Then, $\xi$ is a symmetric reverse $*$ - $n$-derivations.

Lemma (2.8) [11]: Let $\mathcal{R}$ be a prime ring and $\xi: \mathcal{R} \rightarrow \mathcal{R}$ be a derivation such that $a \in \mathcal{R}$. If $a \xi(v)=0$ holds for all $v \in \mathcal{R}$, then either $a=0$ or $\xi=0$.

Lemma (2.9) [14]: Let $\mathcal{R}$ be a $n$ !-torsion free ring and $\lambda \gamma_{1}+\lambda^{2} \gamma_{2}+\ldots+\lambda^{n} \gamma_{n}=0$ where $\gamma_{1}, \gamma_{2}, \ldots, \gamma_{n} \in \mathcal{R}$ with $\lambda=1,2, \ldots, n$. Then $\gamma_{i}=0$, for all $i=1,2, \ldots, n$.

Lemma (2.10) [9]: Let $\mathcal{R}$ be a $n$ !-torsion free $\operatorname{ring}$ and $\lambda \gamma_{1}+\lambda^{2} \gamma_{2}+\ldots+\lambda^{n} \gamma_{n} \in \mathcal{Z}(\mathcal{R})$ where $\gamma_{1}, \gamma_{2}, \ldots, \gamma_{n} \in \mathcal{R}$ with $\lambda=1,2, \ldots, n$. Then $\gamma_{i} \in \mathcal{Z}$, for all $i=1,2, \ldots, n$.

\section{The Main Results}

The commuting and centralizing of symmetric reverse $*$ - $n$-derivations are studied and investigate the commutativity of prime $*$-ring with symmetric reverse $*-n$-derivations that satisfying certain conditions to obtain main results.

In the following results, $\mathcal{U}$ assumed as an admissible Lie ideal of $n$ !-torsion free ring $\mathcal{R}$ with $n \geq 2$.

Theorem (3.1): Let $\mathcal{R}$ be a prime $*$-ring and $\Omega$ : $\mathcal{U}^{n} \rightarrow \mathcal{R}$ be a symmetric reverse $*$ - $n$-derivation associated with involution. If the trace $\delta$ of $\Omega$ satisfies $\left[\delta(v), v^{*}\right]=0$, for all $v \in \mathcal{U}$ then $\Omega\left(v_{1}, v_{2}, \ldots, v_{n}\right)=0$, for all $v_{i} \in \mathcal{U}, i=1,2, \ldots, n$.

\section{Proof:}

$\left[\delta(v), v^{*}\right]=0, \quad \forall v \in \mathcal{U}$

Substituting $v=v+\mu \gamma$ in equation (1) and using it and let $\mu(1 \leq \mu \leq n)$ be any integer, to obtain

$$
\left.0=\left[\delta(v+\mu \gamma), v^{*}+\mu \gamma^{*}\right)\right]
$$

$=\left[\delta(v)+\delta(\mu \gamma)+\sum_{s=1}^{n-1} C_{s} f_{s}(v, \mu \gamma), v^{*}+\mu \gamma^{*}\right]$

$=\mu\left\{\left[\delta(v), \gamma^{*}\right]+\left[c_{1} f_{1}(v, \gamma), v^{*}\right]\right\}+\mu^{2}\left\{\left[c_{2} f_{2}(v, \gamma), v^{*}\right]+\left[c_{1} f_{1}(v, \gamma), \gamma^{*}\right]\right\}+\ldots+\mu^{n}\left\{\left[\delta(\gamma), v^{*}\right]+\left[c_{n-1} f_{n-1}(v, \gamma), \gamma^{*}\right]\right\}$ ... (2)

Applying lemma (2.9) to equation (2), to get

$\left[\delta(v), \gamma^{*}\right]+\left[c_{1} f_{1}(v, \gamma), v^{*}\right]=0$

Replacing $\gamma=2 v \gamma$ in equation (3) then

$0=\left[\delta(v),(2 v \gamma)^{*}\right]+\left[c_{1} f_{1}(v, 2 v \gamma), v^{*}\right]$

$=\left[\delta(v), \gamma^{*}\right] v^{*}+c_{1}\left[f_{1}(v, \gamma), v^{*}\right] v^{*}+c_{1}\left[\gamma, v^{*}\right] \delta(v)+c_{1} \gamma\left[\delta(v), v^{*}\right]$

$=\left\{\left[\delta(v), \gamma^{*}\right]+c_{1}\left[f_{1}(v, \gamma), v^{*}\right]\right\} v^{*}+c_{1}\left[\gamma, v^{*}\right] \delta(v)$ 
By using equation (3) with the equation above to obtain

$c_{1}\left[\gamma, v^{*}\right] \delta(v)=0$

Using $n$ !-torsion freeness, to get

$\left[\gamma, v^{*}\right] \delta(v)=0, \quad \forall v, \gamma \in \mathcal{U}$

Replacing $\gamma=2 \gamma w$ in equation (4) and using it, for all $w \in \mathcal{U}$ then

$0=\left[2 \gamma w, v^{*}\right] \delta(v)$

$=\left[\gamma, v^{*}\right] w \delta(v)$

By using lemma (2.8), that $\gamma \rightarrow\left[\gamma, \alpha^{*}(v)\right]$ is a derivation on $\mathcal{U}$.

Then $\delta(v)=0$

Now, for each value $l=1,2, \ldots, n$, let us denote

$T_{l}(v)=\Omega\left(v, v, \ldots, v_{l+1}, v_{l+2}, \ldots, v_{n}\right)$, where $v, v_{i} \in \mathcal{U}, i=l+1, l+2, \ldots, n$.

$T_{n}(v)=\delta(v)=0$

Let $\eta(1 \leq \eta \leq n)$ be any positive integer. From equation (7) to have

$0=T_{n}\left(\eta v+v_{n}\right)=T_{n}\left(v_{n}\right)+T_{n}(\eta v)+\sum_{l=1}^{n-1} \eta^{l} C_{l} T_{l}(v)=\delta\left(v_{n}\right)+\eta^{n} \delta(v)+\sum_{l=1}^{n-1} \eta^{l} C_{l} T_{l}(v)$

$=\sum_{l=1}^{n-1} \eta^{l} C_{l} T_{l}(v)=\eta^{1} C_{1} T_{1}(v)+\eta^{2} C_{2} T_{2}(v)+\ldots+\eta^{n-1} C_{n-1} T_{n-1}(v)$

Applying lemma (2.9) to equation (8) then

$c_{1} T_{1}(v)=0$ then $T_{1}(v)=0$ which implies that $\Omega\left(v, v_{2}, v_{3}, \ldots, v_{n}\right)=0$

$c_{2} T_{2}(v)=0$ then $T_{2}(v)=0$ which implies that $\Omega\left(v, v, v_{3}, \ldots, v_{n}\right)=0$

$c_{n-1} T_{n-1}(v)=0$ then $T_{n-1}(v)=0$ which implies that $\Omega\left(v, v, v, \ldots, v_{n}\right)=0$

Hence from above we have $T_{n-1}(v)=0$

Again let $\tau(1 \leq \tau \leq n-1)$ be any positive integer. Then from equation (9) to get

$0=T_{n-1}\left(\tau v+v_{n-1}\right)=T_{n-1}(\tau v)+T_{n-1}\left(v_{n-1}\right)+\sum_{t=1}^{n-2} \tau^{t} C_{t} T_{t}(v)$

$=\tau^{1} C_{1} T_{1}(v)+\tau^{2} C_{2} T_{2}(v)+\cdots+\tau^{n-2} C_{n-2} T_{n-2}(v)$

Again applying lemma (2.9) to equation (10) to get

$\Omega\left(v, v, \ldots, v, v_{n-1}, v_{n}\right)=T_{n-2}(v)=0$

Continuing the above process, finally we obtain $T_{1}(v)=0$, then

$\Omega\left(v_{1}, v_{2}, v_{3}, \ldots, v_{n-1}, v_{n}\right)=0$

Replacing $v_{1}=2 v_{1} p_{1}$, where $p_{1} \in \mathcal{U}$ in equation (12) to get

$0=\Omega\left(2 v_{1} p_{1}, v_{2}, v_{3}, \ldots, v_{n-1}, v_{n}\right)=\alpha\left(p_{1}\right) \Omega\left(v_{1}, v_{2}, v_{3}, \ldots, v_{n-1}, v_{n}\right)+v_{1} \Omega\left(p_{1}, v_{2}, v_{3}, \ldots, v_{n-1}, v_{n}\right)=v_{1} \Omega\left(p_{1}, v_{2}, v_{3}, \ldots, v_{n-1}, v_{n}\right)$ ... (13)

Applying lemma (2.8) to equation (13) then 
$\Omega\left(p_{1}, v_{2}, v_{3}, \ldots, v_{n-1}, v_{n}\right)=0, \forall p_{1}, v_{i} \in \mathcal{U}$.

Replacing $v_{2}=v_{2} p_{2}, p_{2} \in \mathcal{U}$ in equation (13) to obtain

$0=\Omega\left(p_{1}, v_{2} p_{2}, v_{3}, \ldots, v_{n-1}, v_{n}\right)=\alpha\left(p_{2}\right) \Omega\left(p_{1}, v_{2}, v_{3}, \ldots, v_{n-1}, v_{n}\right)+v_{2} \Omega\left(p_{1}, p_{2}, \ldots, v_{n-1}, v_{n}\right)=v_{2} \Omega\left(p_{1}, p_{2}, \ldots, v_{n-1}, v_{n}\right)=$ $\Omega\left(p_{1}, p_{2}, \ldots, v_{n-1}, v_{n}\right), \forall p_{1}, p_{2}, v_{i} \in \mathcal{U}$

Repeating the above process we finally obtain $\Omega\left(p_{1}, p_{2}, \ldots, p_{n-1}, p_{n}\right)=0, \forall p_{i} \in \mathcal{U}$.

Theorem (3.2): Let $\mathcal{R}$ be a semiprime $*$-ring and $\Omega: \mathcal{U}^{n} \rightarrow \mathcal{R}$ be a symmetric reverse $*-n$-derivation associated with involution. If the trace $\delta$ of $\Omega$ such that $\delta$ is commuting on $\mathcal{U}$ and $\left[\delta(v), v^{*}\right] \in \mathcal{Z}(\mathcal{R})$, then $\left[\delta(v), v^{*}\right]=0$ for all $v \in \mathcal{U}$.

\section{Proof:}

$\left[\delta(v), v^{*}\right] \in \mathcal{Z}(\mathcal{R}), \quad \forall v \in \mathcal{U}$

Substituting $v=v+\mu \gamma$ in equation (1) and using it and let $\mu(1 \leq \mu \leq n)$ be any integer, then

$Z(\mathcal{R}) \ni\left[\delta(v+\mu \gamma), v^{*}+\mu \gamma^{*}\right]$

$=\left[\delta(v)+\delta(\mu \gamma)+\sum_{s=1}^{n-1} C_{s} f_{s}(v, \mu \gamma), v^{*}+\mu \gamma^{*}\right]$

$=\left[\delta(v), v^{*}\right]+\mu\left\{\left[\delta(v), \gamma^{*}\right]+\left[c_{1} f_{1}(v, \gamma), v^{*}\right]\right\}+\mu^{2}\left\{\left[c_{2} f_{2}(v, \gamma), v^{*}\right]+\left[c_{1} f_{1}(v, \gamma), \gamma^{*}\right]\right\}+\cdots+\mu^{n}\left\{\left[\delta(\gamma), v^{*}\right]+\right.$

$\left.\left[c_{n-1} f_{n-1}(v, y), \gamma^{*}\right]\right\}+\mu^{n+1}\left[\delta(\gamma), \gamma^{*}\right]$

Commuting equation (2) with $\delta(v)$ to get

$\left[\left[\delta(v), v^{*}\right], \delta(v)\right]+\mu\left\{\left[\left[\delta(v), \gamma^{*}\right]+\left[c_{1} f_{1}(v, \gamma), v^{*}\right], \delta(v)\right]\right\}+\mu^{2}\left\{\left[\left[c_{2} f_{2}(v, \gamma), v^{*}\right]\right.\right.$

$\left.\left.\left[c_{1} f_{1}(v, \gamma), \gamma^{*}\right], \delta(v)\right]\right\}+\ldots+\mu^{n}\left\{\left[\left[\delta(\gamma), v^{*}\right]+\left[c_{n-1} f_{n-1}(v, \gamma), \gamma^{*}\right], \delta(v)\right]\right\}+\mu^{n+1}\left[\left[\delta(\gamma), \gamma^{*}\right], \delta(v)\right]=0$

Applying lemma (2.9) to equation (3) to have

$\left[\left[\delta(v), \gamma^{*}\right], \delta(v)\right]+\left[\left[c_{1} f_{1}(v, \gamma), v^{*}\right], \delta(v)\right]=0$

Replacing $\gamma=2 v^{2}$ in equation (4) to get

$0=\left[\left[\delta(v),\left(2 v^{2}\right)^{*}\right], \delta(v)\right]+\left[\left[c_{1} f_{1}\left(v, 2 v^{2}\right), v^{*}\right], \delta(v)\right]$

$=\left[\left[\delta(v), v^{*}\right], \delta(v)\right] v^{*}+\left[\delta(v), v^{*}\right]\left[v^{*}, \delta(v)\right]+\left[v^{*}, \delta(v)\right]\left[\delta(v), v^{*}\right]+v^{*}\left[\left[\delta(v), v^{*}\right], \delta(v)\right]+c_{1}\left[\left[\delta(v), v^{*}\right], \delta(v)\right] v^{*}+$ $c_{1}\left[\delta(v), v^{*}\right]\left[v^{*}, \delta(v)\right]+c_{1}\left[\left[v, v^{*}\right], \delta(v)\right] \delta(v)+c_{1}\left[v, v^{*}\right][\delta(v), \delta(v)]+c_{1}[v, \delta(v)]\left[\delta(v), v^{*}\right]+c_{1} v\left[\left[\delta(v), v^{*}\right], \delta(v)\right]$

$=-\left(c_{1}+2\right)\left[\delta(v), v^{*}\right]^{2}+c_{1}\left[\left[v, v^{*}\right], \delta(v)\right] \delta(v)$

$=-\left(c_{1}+2\right)\left[\delta(v), v^{*}\right]^{2}+\left[[v, \delta(v)], v^{*}\right] \delta(v)$

$=\left(c_{1}+2\right)\left[\delta(v), v^{*}\right]^{2}$

Commuting equation (2) with $v^{*}$ and using lemma (2.9) to get

$\left.0=\left[\left[\delta(v), \gamma^{*}\right], v^{*}\right]+\left[c_{1} f_{1}(v, \gamma), v^{*}\right], v^{*}\right]$

Replacing $\gamma=2 v \gamma$ in equation (6) to obtain

$0=\left[\left[\delta(v),(2 v \gamma)^{*}\right], v^{*}\right]+\left[\left[c_{1} f_{1}(v, 2 v \gamma), v^{*}\right], v^{*}\right]$

$=\left[\left[\delta(v), \gamma^{*}\right], v^{*}\right] v^{*}+\left[\gamma^{*}, v^{*}\right]\left[\delta(v), v^{*}\right]+\gamma^{*}\left[\left[\delta(v), v^{*}\right], v^{*}\right]+c_{1}\left[\left[f_{1}(v, \gamma), v^{*}\right], v^{*}\right] v^{*}+c_{1}\left[\left[\gamma, v^{*}\right], v^{*}\right] \delta(v)+c_{1}\left[\gamma, v^{*}\right]\left[\delta(v), v^{*}\right]+$ $c_{1}\left[\gamma, v^{*}\right]\left[\delta(x), v^{*}\right]+c_{1} \gamma\left[\left[\delta(v), v^{*}\right], v^{*}\right]$

$=\left\{\left[\left[\delta(v), \gamma^{*}\right], v^{*}\right]+c_{1}\left[\left[f_{1}(v, \gamma), v^{*}\right], v^{*}\right]\right\} v^{*}+\left[\gamma^{*}, v^{*}\right]\left[\delta(v), v^{*}\right]+c_{1}\left[\left[\gamma, v^{*}\right], v^{*}\right] \delta(v)+2 c_{1}\left[\gamma, v^{*}\right]\left[\delta(v), v^{*}\right]$ 
By using equation (6) with above equation to get

$\left[\gamma^{*}, v^{*}\right]\left[\delta(v), v^{*}\right]+c_{1}\left[\left[\gamma, v^{*}\right], v^{*}\right] \delta(v)+2 c_{1}\left[\gamma, v^{*}\right]\left[\delta(v), v^{*}\right]=0$

Replacing $\gamma=\delta(v)\left[\delta(v), v^{*}\right]$ in equation (7), to have

$0=\left[\left[\delta(v), v^{*}\right] \delta(v), v^{*}\right]\left[\delta(v), v^{*}\right]+c_{1}\left[\left[\delta(v)\left[\delta(v), v^{*}\right], v^{*}\right], v^{*}\right] \delta(v)+2 c_{1}\left[\delta(v)\left[\delta(v), v^{*}\right], v^{*}\right]\left[\delta(v), v^{*}\right]$

$=\left(2 c_{1}+1\right)\left[\delta(v), v^{*}\right]^{3}$

$=\left(2 c_{1}+1\right)\left[\delta(v), v^{*}\right]^{2} \mathcal{U}\left(2 c_{1}+1\right)\left[\delta(v), v^{*}\right]^{2}$

Since $\mathcal{R}$ is a semiprime, then

$\left(2 c_{1}+1\right)\left[\delta(v), v^{*}\right]^{2}=0$, for all $v \in \mathcal{U}$

Combining equations (5) and (9) to get

$\left[\delta(v), v^{*}\right]^{2}=0$, for all $v \in \mathcal{U}$.

As the center of the semiprime ring contains no non-zero nilpotent elements, then $\left[\delta(v), v^{*}\right]=0$, for all $v \in \mathcal{U}$.

Theorem (3.3): Let $\mathcal{R}$ be a prime $*$-ring and $\Omega: \mathcal{U}^{n} \rightarrow \mathcal{R}$ be a non-zero symmetric reverse $*$ - $n$-derivation associated with involution. If the trace $\delta$ of $\Omega$ is commuting on $\mathcal{U}$ and $\left[\delta(v), v^{*}\right] \in \mathcal{Z}(\mathcal{R})$ for all $v \in \mathcal{U}$, then $\mathcal{U}$ must be commutative.

\section{Proof:}

Suppose that $\mathcal{U}$ is anon commutative prime ring. From Theorem (3.2) we have $\left[\delta(v), v^{*}\right]=0$ for all $v \in \mathcal{U}$. And from Theorem (3.1) we have $\Omega=0$ which it contradiction, hence $U$ is commutative prime ring.

Theorem (3.4): Let $\mathcal{R}$ be a semiprime $*$-ring. If $\mathcal{R}$ admits a symmetric reverse $*-n$-derivation $\xi$ of $\mathcal{R}$, then $\xi$ is a maps from $\mathcal{R}$ to $\mathcal{Z}(\mathcal{R})$.

Proof: By hypothesis

$\xi\left(v \gamma, v_{2}, \ldots, v_{n}\right)=\xi\left(\gamma, v_{2}, \ldots, v_{n}\right) v^{*}+\gamma \xi\left(v, v_{2}, \ldots, v_{n}\right)$

Let $\gamma=\gamma z$ in equation (1) to get

$\xi\left(v \gamma z, v_{2}, \ldots, v_{n}\right)=\xi\left(\gamma z, v_{2}, \ldots, v_{n}\right) v^{*}+\gamma z \xi\left(v, v_{2}, \ldots, v_{n}\right)$

$=\xi\left(z, v_{2}, \ldots, v_{n}\right) \gamma^{*} v^{*}+z \xi\left(\gamma, v_{2}, \ldots, v_{n}\right) v^{*}+\gamma z \xi\left(v, v_{2}, \ldots, v_{n}\right)$, for all $v, \gamma, z, v_{2}, \ldots, v_{n} \in \mathcal{R} \ldots$

Also, $\xi\left(v \gamma z, v_{2}, \ldots, v_{n}\right)=\xi\left(z, v_{2}, \ldots, v_{n}\right) \gamma^{*} v^{*}+z \xi\left(v \gamma, v_{2}, \ldots, v_{n}\right)$

$=\xi\left(z, v_{2}, \ldots, v_{n}\right) \gamma^{*} v^{*}+z \xi\left(\gamma, v_{2}, \ldots, v_{n}\right) v^{*}+z \gamma \xi\left(v, v_{2}, \ldots, v_{n}\right)$

Comparing equations (2) and (3) to have

$[\gamma, z] \xi\left(v, v_{2}, \ldots, v_{n}\right)=0$

Replacing $\gamma=\xi\left(v, v_{2}, \ldots, v_{n}\right) \gamma$ in equation (4) and using it then

$\left[\xi\left(v, v_{2}, \ldots, v_{n}\right), z\right] \gamma \xi\left(v, v_{2}, \ldots, v_{n}\right)=0$ 
Let $\gamma=\gamma z$ in equation (5) to have

$\left[\xi\left(v, v_{2}, \ldots, v_{n}\right), z\right] \gamma z \xi\left(v, v_{2}, \ldots, v_{n}\right)=0$

Now, multiplying equation (5) from the right side by $z$, to have

$\left[\xi\left(v, v_{2}, \ldots, v_{n}\right), z\right] \gamma \xi\left(v, v_{2}, \ldots, v_{n}\right) z=0$

Comparing equations (6) and (7) then

$\left[\xi\left(v, v_{2}, \ldots, v_{n}\right), z\right] \gamma\left[\xi\left(v, v_{2}, \ldots, v_{n}\right), z\right]=0$, hence $\left[\xi\left(v, v_{2}, \ldots, v_{n}\right), z\right] \mathcal{R}\left[\xi\left(v, v_{2}, \ldots, v_{n}\right), z\right]=0$. Since $\mathcal{R}$ is semiprime then $\left[\xi\left(v, v_{2}, \ldots, v_{n}\right), z\right]=0$ for all $v, z, v_{2}, \ldots, v_{n} \in \mathcal{R}$ and then $\xi$ is a map from $\mathcal{R}$ into $\mathcal{Z}(\mathcal{R})$.

Theorem (3.5): Let $\mathcal{R}$ be a prime $*$-ring. If $\mathcal{R}$ admits a symmetric reverse $*-n$-derivation $\xi$ of $\mathcal{R}$ such that $\xi\left(v, v_{2}, \ldots, v_{n}\right) \neq v$ and $\xi\left(v \gamma, v_{2}, \ldots, v_{n}\right)=\xi\left(v, v_{2}, \ldots, v_{n}\right) \xi\left(\gamma, v_{2}, \ldots, v_{n}\right)$ for all $v, \gamma, v_{2}, \ldots, v_{n} \in \mathcal{R}$ then $\xi=0$.

Proof: By hypothesis

$\xi\left(v \gamma, v_{2}, \ldots, v_{n}\right)=\xi\left(\gamma, v_{2}, \ldots, v_{n}\right) v^{*}+\gamma \xi\left(v, v_{2}, \ldots, v_{n}\right)=\xi\left(v, v_{2}, \ldots, v_{n}\right) \xi\left(\gamma, v_{2}, \ldots, v_{n}\right)$

Let $\gamma=z \gamma$ in equation (1) to get

$\xi\left(z, v_{2}, \ldots, v_{n}\right) \xi\left(\gamma, v_{2}, \ldots, v_{n}\right) v^{*}+z \gamma \xi\left(v, v_{2}, \ldots, v_{n}\right)=\xi\left(v, v_{2}, \ldots, v_{n}\right) \quad \xi\left(z, v_{2}, \ldots, v_{n}\right) \xi\left(\gamma, v_{2}, \ldots, v_{n}\right)=$ $\left.\xi\left(v z, v_{2}, \ldots, v_{n}\right) \xi\left(\gamma, v_{2}, \ldots, v_{n}\right)=\left\{\xi\left(z, v_{2}, \ldots, v_{n}\right) v^{*}+z \xi\left(v, v_{2}, \ldots, v_{n}\right)\right\} \xi\left(\gamma, v_{2}, \ldots, v_{n}\right)\right\}$

This implies that

$\xi\left(z, v_{2}, \ldots, v_{n}\right)\left[\xi\left(\gamma, v_{2}, \ldots, v_{n}\right), v^{*}\right]+z \xi\left(v, v_{2}, \ldots, v_{n}\right)\left(\gamma-\xi\left(\gamma, v_{2}, \ldots, v_{n}\right)\right)=0$

By theorem (3.4) the above equation becomes

$z \xi\left(v, v_{2}, \ldots, v_{n}\right)\left(\gamma-\xi\left(\gamma, v_{2}, \ldots, v_{n}\right)\right)=0$

Hence, $\xi\left(v, v_{2}, \ldots, v_{n}\right) z\left(\gamma-\xi\left(\gamma, v_{2}, \ldots, v_{n}\right)\right)=0$. We can written as $\xi\left(v, v_{2}, \ldots, v_{n}\right) \mathcal{R}\left(\gamma-\xi\left(\gamma, v_{2}, \ldots, v_{n}\right)\right)=0$. Since $\mathcal{R}$ is prime then either $\xi\left(v, v_{2}, \ldots, v_{n}\right)=0$ or $\left(\gamma-\xi\left(\gamma, v_{2}, \ldots, v_{n}\right)\right)=0$, but $\xi\left(\gamma, v_{2}, \ldots, v_{n}\right) \neq \gamma$, then $\xi\left(v, v_{2}, \ldots, v_{n}\right)=0$ for all $v, v_{2}, \ldots, v_{n} \in \mathcal{R}$.

Theorem (3.6): Let $\mathcal{R}$ be a prime *-ring. If $\mathcal{R}$ admits a reverse $*-n$-derivation $\xi$ of $\mathcal{R}$ such that $\xi\left(v, v_{2}, \ldots, v_{n}\right) \neq v^{*}$ and $\xi\left(v \gamma, v_{2}, \ldots, v_{n}\right)=\xi\left(\gamma, v_{2}, \ldots, v_{n}\right) \xi\left(v, v_{2}, \ldots, v_{n}\right)$ for all $v, \gamma, v_{2}, \ldots, v_{n} \in \mathcal{R}$ then $\xi=0$.

Proof: By hypothesis

$\xi\left(v \gamma, v_{2}, \ldots, v_{n}\right)=\xi\left(\gamma, v_{2}, \ldots, v_{n}\right) v^{*}+\gamma \xi\left(v, v_{2}, \ldots, v_{n}\right)=\xi\left(\gamma, v_{2}, \ldots, v_{n}\right) \xi\left(v, v_{2}, \ldots, v_{n}\right)$

Replacing $v=v \gamma$ in equation (1) to get

$\xi\left(\gamma, v_{2}, \ldots, v_{n}\right) \gamma^{*} v^{*}+\gamma \xi\left(\gamma, v_{2}, \ldots, v_{n}\right) \xi\left(v, v_{2}, \ldots, v_{n}\right)=\xi\left(\gamma, v_{2}, \ldots, v_{n}\right) \xi\left(v \gamma, v_{2}, \ldots, v_{n}\right)=\xi\left(\gamma, v_{2}, \ldots, v_{n}\right)\left\{\xi\left(\gamma, v_{2}, \ldots, v_{n}\right) v^{*}+\right.$ $\left.\gamma \xi\left(v, v_{2}, \ldots, v_{n}\right)\right\}$

By theorem (3.4) then

$\xi\left(\gamma, v_{2}, \ldots, v_{n}\right) \gamma^{*} v^{*}-\xi\left(\gamma, v_{2}, \ldots, v_{n}\right) \xi\left(\gamma, v_{2}, \ldots, v_{n}\right) v^{*}=0$

$\xi\left(\gamma, v_{2}, \ldots, v_{n}\right)\left(\gamma^{*}-\xi\left(\gamma, v_{2}, \ldots, v_{n}\right)\right) v^{*}=0$

Hence $\xi\left(\gamma, v_{2}, \ldots, v_{n}\right) v^{*}\left(\gamma^{*}-\xi\left(\gamma, v_{2}, \ldots, v_{n}\right)\right)=0$

We can written as $\xi\left(\gamma, v_{2}, \ldots, v_{n}\right) \mathcal{R}\left(\gamma^{*}-\xi\left(\gamma, v_{2}, \ldots, v_{n}\right)\right)=0$. Since $\mathcal{R}$ is prime then either $\xi\left(\gamma, v_{2}, \ldots, v_{n}\right)=0$ or $\left(\gamma^{*}-\xi\left(\gamma, v_{2}, \ldots, v_{n}\right)\right)=0$, but $\xi\left(\gamma, v_{2}, \ldots, v_{n}\right) \neq \gamma^{*}$, then we have that $\xi\left(\gamma, v_{2}, \ldots, v_{n}\right)=0$ for all $\gamma, v_{2}, \ldots, v_{n} \in \mathcal{R}$. 
Theorem (3.7): Let $\mathcal{R}$ be a prime $*$-ring and $a \in \mathcal{R}$. If $\mathcal{R}$ admits a symmetric reverse $*$ - $n$-derivation $\xi$ of $\mathcal{R}$ and $\left[\xi\left(v, v_{2}, \ldots, v_{n}\right), a\right]=0$, then $\xi(a)=0$ or $a \in \mathcal{Z}(\mathcal{R})$.

Proof: By hypothesis

$\left[\xi\left(v \gamma, v_{2}, \ldots, v_{n}\right), a\right]=0$, for all $v, \gamma, v_{2}, \ldots, v_{n} \in \mathcal{R}$

That is

$\left[\xi\left(\gamma, v_{2}, \ldots, v_{n}\right) v^{*}+\gamma \xi\left(v, v_{2}, \ldots, v_{n}\right), a\right]=0$

Hence, $\xi\left(\gamma, v_{2}, \ldots, v_{n}\right)\left[v^{*}, a\right]+[\gamma, a] \xi\left(v, v_{2}, \ldots, v_{n}\right)=0$

Replacing $\gamma=a$ and $v^{*}=v$ in equation (2) to get

$\xi\left(a, v_{2}, \ldots, v_{n}\right)[v, a]=0$

Replacing $v=v \gamma$ in equation (3) and using it then

$\xi\left(a, v_{2}, \ldots, v_{n}\right) v[\gamma, a]=0$

This implies that $\xi\left(a, v_{2}, \ldots, v_{n}\right) \mathcal{R}[\gamma, a]=0$. Since $\mathcal{R}$ is prime then $\xi d\left(a, v_{2}, \ldots, v_{n}\right)=0$ for all $a, v_{2}, \ldots, v_{n} \in \mathcal{R}$ or $a \in \mathcal{Z}(\mathcal{R})$.

Theorem (3.8): Let $\mathcal{R}$ be a semiprime $*$-ring. If $\mathcal{R}$ admits a symmetric reverse $*-n$-derivation $\mathrm{d}$ of $\mathcal{R}$ then $\left[\xi\left(v, v_{2}, \ldots, v_{n}\right), z\right]=0$ for all $v, z, v_{2}, \ldots, v_{n} \in \mathcal{R}$.

Proof: By hypothesis

$\xi\left(v \gamma, v_{2}, \ldots, v_{n}\right)=\xi\left(\gamma, v_{2}, \ldots, v_{n}\right) v^{*}+\gamma \xi\left(v, v_{2}, \ldots, v_{n}\right)$

Substituting $v=v z$ in equation (1) to get

$\xi\left(v z \gamma, v_{2}, \ldots, v_{n}\right)=\xi\left(\gamma, v_{2}, \ldots, v_{n}\right)(v z)^{*}+\gamma \xi\left(v z, v_{2}, \ldots, v_{n}\right)$

$=\xi\left(\gamma, v_{2}, \ldots, v_{n}\right) z^{*} v^{*}+\gamma \xi\left(z, v_{2}, \ldots, v_{n}\right) v^{*}+\gamma z \xi\left(v, v_{2}, \ldots, v_{n}\right)$

Also $\xi\left(v z \gamma, v_{2}, \ldots, v_{n}\right)=\xi\left(z \gamma, v_{2}, \ldots, v_{n}\right) v^{*}+z \gamma \xi\left(v, v_{2}, \ldots, v_{n}\right)$

$=\xi\left(\gamma, v_{2}, \ldots, v_{n}\right) z^{*} v^{*}+\gamma \xi\left(z, v_{2}, \ldots, v_{n}\right) v^{*}+z \gamma \xi\left(v, v_{2}, \ldots, v_{n}\right)$

Comparing equations (2) and (3) to get

$[\gamma, z] \xi\left(v, v_{2}, \ldots, v_{n}\right)=0$

Replacing $\gamma=\xi\left(v, v_{2}, \ldots, v_{n}\right) \gamma$ in equation (4) and using it then

$\left[\xi\left(v, v_{2}, \ldots, v_{n}\right), z\right] \gamma \xi\left(v, v_{2}, \ldots, v_{n}\right)=0$

Let $\gamma=\gamma z$ in equation (5) to have

$\left[\xi\left(v, v_{2}, \ldots, v_{n}\right), z\right] \gamma z \xi\left(v, v_{2}, \ldots, v_{n}\right)=0$

Now, multiplying equation (5) from the right side by $z$ to have

$\left[\xi\left(v, v_{2}, \ldots, v_{n}\right), z\right] \gamma \xi\left(v, v_{2}, \ldots, v_{n}\right) z=0$

Comparing equations (6) and (7) then 
$\left[\xi\left(v, v_{2}, \ldots, v_{n}\right), z\right] \gamma\left[\xi\left(v, v_{2}, \ldots, v_{n}\right), z\right]=0$

Hence, $\left[\xi\left(v, v_{2}, \ldots, v_{n}\right), z\right] \mathcal{R}\left[\xi\left(v, v_{2}, \ldots, v_{n}\right), z\right]=0$. Since $\mathcal{R}$ is semiprime then $\left[\xi\left(v, v_{2}, \ldots, v_{n}\right), z\right]=0$, for all $v, z, v_{2}, \ldots, v_{n} \in \mathcal{R}$.

Theorem (3.9): Let $\mathcal{R}$ be a prime $*$-ring. If $\mathcal{R}$ admits a symmetric reverse $*-n$-derivation $\xi$ of $\mathcal{R}$ such that $\xi\left([v, \gamma], v_{2}, \ldots, v_{n}\right)=0$ for all $v, \gamma, v_{2}, \ldots, v_{n} \in \mathcal{R}$ then $\xi=0$ or $\mathcal{R}$ is commutative.

Proof: By hypothesis

$\xi\left([v, \gamma], v_{2}, \ldots, v_{n}\right)=0$

Let $v=\gamma v$ in equation (1) and using it then

$[v, \gamma] \xi\left(\gamma, v_{2}, \ldots, v_{n}\right)=0$

Replacing $v=v z$ in equation (2) to have

$[v, \gamma] z \xi\left(\gamma, v_{2}, \ldots, v_{n}\right)+v[z, \gamma] \xi\left(\gamma, v_{2}, \ldots, v_{n}\right)=0$

By using equation (2) the above equation becomes

$[v, \gamma] z \xi\left(\gamma, v_{2}, \ldots, v_{n}\right)=0$

This implies that $[v, \gamma] \mathcal{R} \xi\left(\gamma, v_{2}, \ldots, v_{n}\right)=0$. Since $\mathcal{R}$ is prime then $[v, \gamma]=0$ and that means $\mathcal{R}$ is commutative, or $\xi\left(\gamma, v_{2}, \ldots, v_{n}\right)=0$ for all $\gamma, v_{2}, \ldots, v_{n} \in \mathcal{R}$.

Theorem (3.10): Let $\mathcal{R}$ be a prime $*$-ring. If $\mathcal{R}$ admits a symmetric reverse $*$ - $n$-derivation $\xi$ of $\mathcal{R}$ such that $\xi\left((v \circ \gamma), v_{2}, \ldots, v_{n}\right)=0$ for all $v, \gamma, v_{2}, \ldots, v_{n} \in \mathcal{R}$ then $\xi=0$ or $\mathcal{R}$ is commutative.

Proof: By hypothesis

$\xi\left((v \circ \gamma), v_{2}, \ldots, v_{n}\right)=0$

Let $v=\gamma v$ in equation (1) and using it then

$(v \circ \gamma) \xi\left(\gamma, v_{2}, \ldots, v_{n}\right)=0$

Replacing $v=s v$ in equation (2) to have

$(s \circ \gamma) v \xi\left(\gamma, v_{2}, \ldots, v_{n}\right)=0$

Hence, $(s \circ \gamma) \mathcal{R} \xi\left(\gamma, v_{2}, \ldots, v_{n}\right)=$. Since $\mathcal{R}$ is prime then $(s \circ \gamma)=0$, replace $s=s z$ to get $s[z, \gamma]=0$. Now let $s=v s$ then $v s[z, \gamma]=0$, that $v \mathcal{R}[z, \gamma]=0$ for $0 \neq v \in \mathcal{R}$ and since $\mathcal{R}$ is prime then $\mathcal{R}$ is commutative, or $\xi\left(\gamma, v_{2}, \ldots, v_{n}\right)=0$ for all $\gamma, v_{2}, \ldots, v_{n} \in \mathcal{R}$.

Theorem (3.11): Let $\mathcal{R}$ be a prime $*$-ring. If $\mathcal{R}$ admits a symmetric reverse $*$ - $n$-derivation $\xi$ of $\mathcal{R}$ such that $\xi\left(v, v_{2}, \ldots, v_{n}\right) \circ \gamma=0$ for all $v, \gamma, v_{2}, \ldots, v_{n} \in \mathcal{R}$ then $\xi=0$ or $\mathcal{R}$ is commutative.

Proof: By hypothesis

$\xi\left(v, v_{2}, \ldots, v_{n}\right) \circ \gamma=0$

Replacing $v=z v$ in equation (1) and using it then

$\xi\left(v, v_{2}, \ldots, v_{n}\right)\left[z^{*}, \gamma\right]-[v, \gamma] \xi\left(z, v_{2}, \ldots, v_{n}\right)=0$ 
Let $v=\gamma$ and $z^{*}=z$ in equation (2) to get

$\xi\left(\gamma, v_{2}, \ldots, v_{n}\right)[z, \gamma]=0$

Replacing $z=z v$ in equation (3) and using it then

$\xi\left(\gamma, v_{2}, \ldots, v_{n}\right) z[v, \gamma]=0$, for all $v, \gamma, z, v_{2}, \ldots, v_{n} \in \mathcal{R}$

This implies that $\xi\left(\gamma, v_{2}, \ldots, v_{n}\right) \mathcal{R}[v, \gamma]=0$, since $\mathcal{R}$ is prime then $\xi\left(\gamma, v_{2}, \ldots, v_{n}\right)=0$ for all $\gamma, v_{2}, \ldots, v_{n} \in \mathcal{R}$ or $\mathcal{R}$ is commutative.

\section{References}

[1] M.Ashraf and M. A. siddeeque, "On *-n-Derivations in Rings with Involution", Georgian Math. J., 22(2015), No. 1, 9-18.

[2] M. Bresar, and J. Vukman, "On Some Additive Mappings in Rings with involution", Aequationes Math., 38(1989), 178-185.

[3] M. Bresar, and J. Vukman, "On Left Derivations and Related Mappings", Proc. Amer. Math. Soc., 110(1990), No. 1, 7-16.

[4] A. K. Faraj, and S. J. Shareef, "Jordan Permuting 3-Derivations of Prime Rings", Iraqi Journal of Science, 58(2017), No. 2A, 687-693.

[5] A. K. Faraj, and S. J. Shareef, " On Generalized Permuting Left 3-Derivations of Prime Rings", Engineering and Technology Journal, 35(2017), No. 1, Part B.

[6] A. Fosner, "Prime and Semiprime Rings with Symmetric Skew 3-Derivations", Aequat. Math., 87(2014), 191-200.

[7] I.N. Herstein, "Topics in Ring Theory", the University of Chicago Press, Chicago, 1969.

[8] K. H. Kim, and Y. H. Lee, "A Note on *-Derivation of Prime *-Rings", International Mathematical Forum, 12(2017), No. 8, 391-398.

[9] K. H. Park, "On Prime and Semiprime Rings with Symmetric n-Derivations", J.Chungcheong Math. Soc., 22(2009), No. 3, 451-458.

[10] C.J. Reddy, S. V. Kumar, and S. M. Rao, "Symmetric Skew 4- Derivations on Prime Rings", Global Jr. of pure and appl. Math., 12(2016), No. 1, 1013-1018.

[11] E. C. Posner, "Derivations in Prime Rings", proc. Amer. Math. Soc., 8(1957), 1093 - 1100.

[12] B.Satyanarayana, and M. Mastan, "Symmetric Skew Reverse n-Derivation on Prime Rings and Semiprime rings", Int. Journal of Math. Trends and Technology, 47(2017), No. 2, 81-86.

[13] M. Samman, and N. Alyamani, "Derivations and Reverse Derivations in Semiprime Rings", Int. Math. Forum, 2(2007), No. 39, 1895-1902.

[14] V. K. Yadav, and R. K. Sharma, "Skew n-Derivations on Prime and Semiprime Rings", Ann Univ Ferrara, 2016, Sep. 1-12.

[15] U. K. Sharma, and S. Kumar, "On generalized *-n-Derivation in *-Ring", Global Journal of Pure and Applied Math., 13(2017), No. 10, 7561-7572. 